\title{
Microscale study of frictional properties of graphene in ultra high vacuum
}

\author{
Diego MARCHETTO ${ }^{1, \dagger}$, Tim FESER ${ }^{2}$, Martin DIENWIEBEL ${ }^{1,2^{*}}$ \\ ${ }^{1}$ Fraunhofer-Institute for Mechanics of Materials, Microtribology Center $\mu$ TC, Wöhlerstr. 11, 79108 Freiburg, Germany \\ ${ }^{2}$ Karlsruhe Institute of Technology, Institute for Applied Materials-Computational Materials Science IAM-CMS, Kaiserstr. 12, 76131 \\ Karlsruhe, Germany \\ ${ }^{\dagger}$ Present adress: Università di Modena e Reggio Emilia,CNR-Istituto Nanoscienze S3, Dipartimento di Scienze Fisiche, Informatiche e \\ Matematiche, Via Campi, 213/a, 41125 Modena, Italy
}

Received: 12 December 2014 / Revised: 10 February 2015 / Accepted: 24 March 2015

(C) The author(s) 2015. This article is published with open access at Springerlink.com

\begin{abstract}
We report on the frictional properties of epitaxial graphene on $\mathrm{SiC}$ in ultra high vacuum. Measurements have been performed using a microtribometer in the load regime of 0.5 to $1 \mathrm{mN}$. We observed that a ruby sphere sliding against graphene results in very low friction coefficients ranging from 0.02 to 0.05 . The friction and also the stability of the graphene layer is higher than that under similar conditions in ambient conditions. The friction shows a load dependence. Finally it was found that graphene masks the frictional anisotropy which was observed on the SiC surface.
\end{abstract}

Keywords: graphene; $\mathrm{SiC}$; friction; vacuum; microtribology; anisotropy

\section{Introduction}

The reduction of friction and wear is a very active field of research both at the macro and at the micro-nanoscale [1]. A traditional way employed in mechanical engineering to reduce friction and wear is to apply liquid or a solid lubricant but a lubricant used at the macroscale will hardly be useful at the micro or the nanoscale. So the need for a specificity of the lubricant or lubrication technique is still a big issue in the tribology of micro- and nano-positioning as well as in micro-electromechanical systems (MEMS). Therefore the goal remains to avoid failures of components by means of coatings, lubricants or surface alteration. Although many studies have already been published the two worlds are still far from being connected.

The dependence of tribological mechanisms on the

* Corresponding author: Martin DIENWIEBEL.

E-mail: martin.dienwiebel@kit.edu, martin.dienwiebel@iwm.fraunhofer.de environmental conditions complicates the situation even more $[2,3]$. Lubrication of sliding surfaces in vacuum (i.e., in space or in vacuum chambers) requires a completely different approach. Liquid lubricants or vapour lubrication cannot be used without constraints. On the other hand layered materials like graphite and $\mathrm{MoS}_{2}$ can be exceptional lubricants if used in the right environment [4-7]. Graphite is a good lubricant in air but is known to be a poor one in vacuum [4]. $\mathrm{MoS}_{2}$ is used in space applications for its good properties in vacuum and dry atmosphere but it is inadvisable in humid environment unless it is doped with metals or nanocomposites [5-7].

The search for a new material that can be a good lubricant at different scales and in different applications and environments is therefore quite active. In this context the studies on graphene seem to show promising results. In the last few years graphene has been studied intensively in different fields of science and technology because of its physical and mechanical properties [8-11]. Also tribological properties of graphene have been investigated in several studies at the 
macro, micro and the nanoscale [12-19]. The frictional behavior of single and double layer graphene has also been investigated theoretically [20]. Except for nanoscale experiments these studies have been performed in ambient conditions at atmospheric pressure. In a previous study, we also showed that graphene epitaxially grown on $\mathrm{SiC}$ reduces the friction coefficient $\mu$ about 5 times with respect to the substrate and it is lower compared to graphite [21]. These results were obtained in ambient conditions and at room temperature. Since graphene is the basic building block of graphite it is interesting to also investigate a possible environmental dependence of the friction coefficient of this material. Lee et al. has shown that the friction coefficient of exfoliated graphene in different environments measured by friction force microscopy (FFM) drops when the material is measured in dry environment [16]. However to our knowledge the microscale friction of epitaxial graphene in ultra high vacuum has not been investigated up to now.

In this work we present friction experiments using a homebuilt ultra-high vacuum (UHV) microtribometer on epitaxially grown graphene [22]. We show that unlike bulk graphite, few-layer graphene can result in a significant reduction of friction in vacuum and therefore might allow the use of this material for space applications.

\section{Experimental details}

\subsection{Graphene samples}

For the reported tests a commercial SiC-6H (0001) (Si face, Si crystal AG, Erlangen, Germany) surface prepared by hydrogen etching was used [21]. Figure 1 depicts a topography image measured by atomic force microscopy (AFM) of the substrate. It shows that the surface has atomically flat terraces extending for several micrometers. The height of the steps is a multiple of half of the unit cell of SiC-H (see Fig. 1(a)).

A graphene sample was received from the group of Thomas Seyller, University of Erlangen (now Chemnitz), that has been epitaxially grown on the Siterminated $\mathrm{SiC}$ (0001) surface. The layer was obtained by thermal decomposition of the SiC surface as decribed in Emtsev et al. [23]. Figure 1(b) shows the surface of the graphene layer. The atomically flat terrace
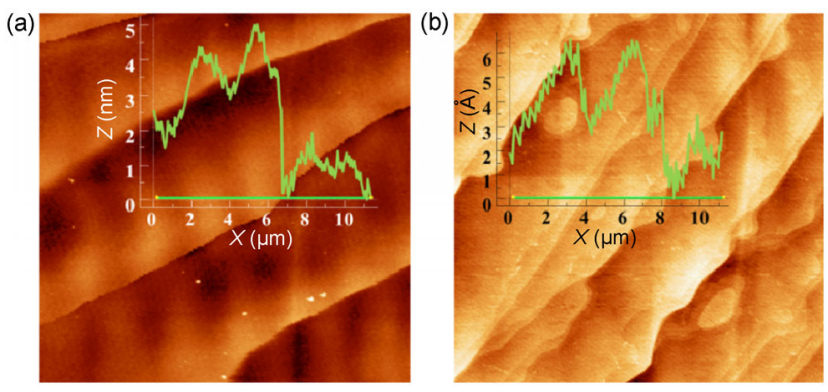

Fig. 1 (a) AFM topographic image of a $\mathrm{SiC}$ (0001) surface (frame size $20 \mu \mathrm{m}$ by $20 \mu \mathrm{m})$. (b) graphene layer growth on $\mathrm{SiC}(0001)$ surface (frame size $20 \mu \mathrm{m}$ by $20 \mu \mathrm{m}$ ) measured by means of AFM (PI Topometrix Explorer with commercial Si tip). The profiles reported both in (a) and (b) show the height of steps. On SiC steps range from $0.75 \mathrm{~nm}$ (half the unit cell of $\mathrm{SiC}-\mathrm{H}$ ) to a few $\mathrm{nm}$ while on graphene steps are about $1 \mathrm{~nm}$.

structure of $\mathrm{SiC}$ is still present; the main difference with the substrate structure is the curved profile of steps. This is probably due to the presence of a second graphene layer along the steps. According to Emtsev et al., the growth process starts at the edge of steps and when a layer is complete a second one is already growing beneath the first [23]. The graphene growth procedure is optimized in order to obtain a single layer on the terraces and can be precisely controlled by the deposition time [23].

Besides the graphene single and bilayer at the step edges all surfaces prepared with this technique exhibit a carbon-rich buffer interlayer between $\mathrm{SiC}$ surface and graphene layer. This interface layer has a graphitic $6 \sqrt{3} \times 6 \sqrt{3}$ structure and it is covalently bound to the $\mathrm{SiC}$ substrate [23]. The graphene layer on top of the interface layer has no covalent bonds to the substrate [24]. Since the interaction between graphene and solvents is not completely clear the samples were not cleaned. Previously we also found no evidence of contamination along several weeks of tests in air [21].

\subsection{Counter face and tribometer set up}

As counter face we used a commercially available ruby ball with a diameter of $500 \mu \mathrm{m}$ (www.spherotech.com) that was also used in previous experiments in air. The RMS roughness of the spheres was previously measured to be $11 \mathrm{~nm}$ on a scale of $5 \mu \mathrm{m}$ leading to a multiple asperities contact as confirmed by Wählisch et al. [25].

Friction experiments have been performed by means of a custom made UHV microtribometer. Details of 
the instruments are described in Ref. [22]. The measurements were performed with a constant applied load and a sliding length of $100 \mu \mathrm{m}$. Since each measurement consisted of a sequence of reciprocating cycles the friction force $F_{\mathrm{F}}$ was determined as the average of the lateral force over one complete cycle $F_{\mathrm{F}}=\left(F_{\text {trace }}-F_{\text {retrace }}\right) / 2$. Data from the beginning and from the end of the track related to the transition from static to kinetic friction were omitted.

Normal loads and speed for these experiments are summarized in Table 1 . We used normal loads of 0.5 and $1.0 \mathrm{mN}$ and speeds of 30 and $50 \mu \mathrm{m} / \mathrm{s}$ for this study.

The residual pressure in the UHV chamber during experiment was approx. $10^{-7} \mathrm{~Pa}$. By rotating the sample inside the chamber we investigated the dependence of friction on the sliding direction (friction anisotropy) as reported below.

Table 1 Load and speed conditions of performed tests.

\begin{tabular}{cc}
\hline \multicolumn{2}{c}{ Friction test conditions } \\
\hline Load $(\mathrm{mN})$ & Speed $(\mu \mathrm{m} / \mathrm{s})$ \\
\hline 0.5 & 30 \\
1.0 & 50 \\
\hline
\end{tabular}

\section{Results}

\subsection{Friction as a function of load on graphene and on $\mathrm{SiC}$}

Friction data obtained from the experiments are plotted in Fig. 2. The plotted values are the average of all the results obtained at the different loads during several experiments. The error bars represent the deviations of the friction coefficient values between the tests. The measured friction coefficients as a function of sliding cycles and normal load for $\mathrm{SiC}$ and graphene are plotted in Fig. 2. The friction of the ruby sphere sliding on graphene in vacuum is about 5 to 8 times lower than that measured on a $\mathrm{SiC}$ single crystal. The friction coefficient of SiC strongly depends on the applied load and it increases during the tests. As visible in Fig. 2 the friction coefficient decreases from 0.42 to 0.35 at a load of $0.5 \mathrm{mN}$ and from 0.30 to 0.22 at a load of $1 \mathrm{mN}$. The change is quite fast in the first 40 cycles but after this first period of sliding $\mu$ decreases at a lower rate (asymptotic behaviour).

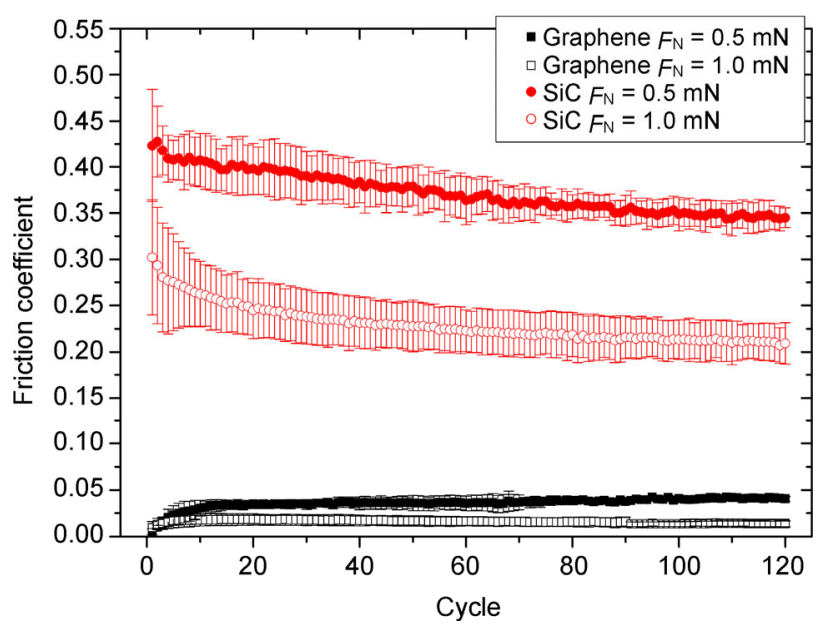

Fig. 2 Evolution of the friction coefficient on $\mathrm{SiC}$ (red) and graphene (black) as function of cycle number for two applied loads 0.5 to $1 \mathrm{mN}$. Each data point was calculated by averaging single friction values obtained during several tests at the different loads. The error bars represent the standard deviation of the values. The friction coefficient $\mu$ is correlated with the applied load. Indeed both $\mathrm{SiC}$ and graphene (with a smaller rate though) show higher friction coefficient at lower applied load.

Differently from Fig. 2, the values reported in Fig. 3(a) were obtained during each test, i.e., not averaged over several tests. The friction coefficient was determined as the average of the lateral force over one complete sliding cycle divided by the applied load following the same procedure used in our previous work [21]. A section of $10 \mu \mathrm{m}$ of data has been cut off at the beginning and at the end of each track in order to omit data from the transition from static to kinetic friction. In this graph a similar load dependency of SiC can be seen for graphene (Fig. 3(a)). The friction coefficient of graphene obtained at $0.5 \mathrm{mN}$ of applied load is about 0.04 and it is higher than that obtained at $1.0 \mathrm{mN}$ which is 0.015 . Although both samples show the same load dependency, the evolution of the friction coefficient of graphene is completely different. In this case $\mu$ starts at values of about 0.005 and rises quickly during the first 10 to 20 cycles to about 0.015 at a load of $1 \mathrm{mN}$ and to 0.035 at a load of $0.5 \mathrm{mN}$. After the first running-in period at $F_{\mathrm{N}}=1 \mathrm{mN}$ the friction coefficient $\mu$ tends to decrease to a slightly lower value and after 80 cycles it stabilizes. This long term oscillation is not visible at $F_{\mathrm{N}}=0.5 \mathrm{mN}$. Instead at this load $\mu$ reaches a "plateau" after 20 cycles and it stays stable for the remainder of the experiment.

The difference between values of $\mu$ on graphene 
obtained in UHV and those obtained in air [21] is shown by the area marked by dashed lines in Fig. 3(a). The friction coefficient measured at $F_{\mathrm{N}}=1 \mathrm{mN}$ in ultra-high vacuum is significantly lower than that measured in air, which is quite surprising. The largest difference is found during the first cycle of each experiment. Indeed, independent of the applied load the tests show a friction coefficient of about 0.005 . This value is 4 times lower than those obtained in air $\left(\mu_{\text {Air }}=0.02\right)$ [21].

Following the approach reported in our previous paper we calculated so-called "triboscopy images" of the friction data (Fig. 3(b)). This represents the spatial variation of the friction coefficient along the sliding track as a function of cycle number. As already seen
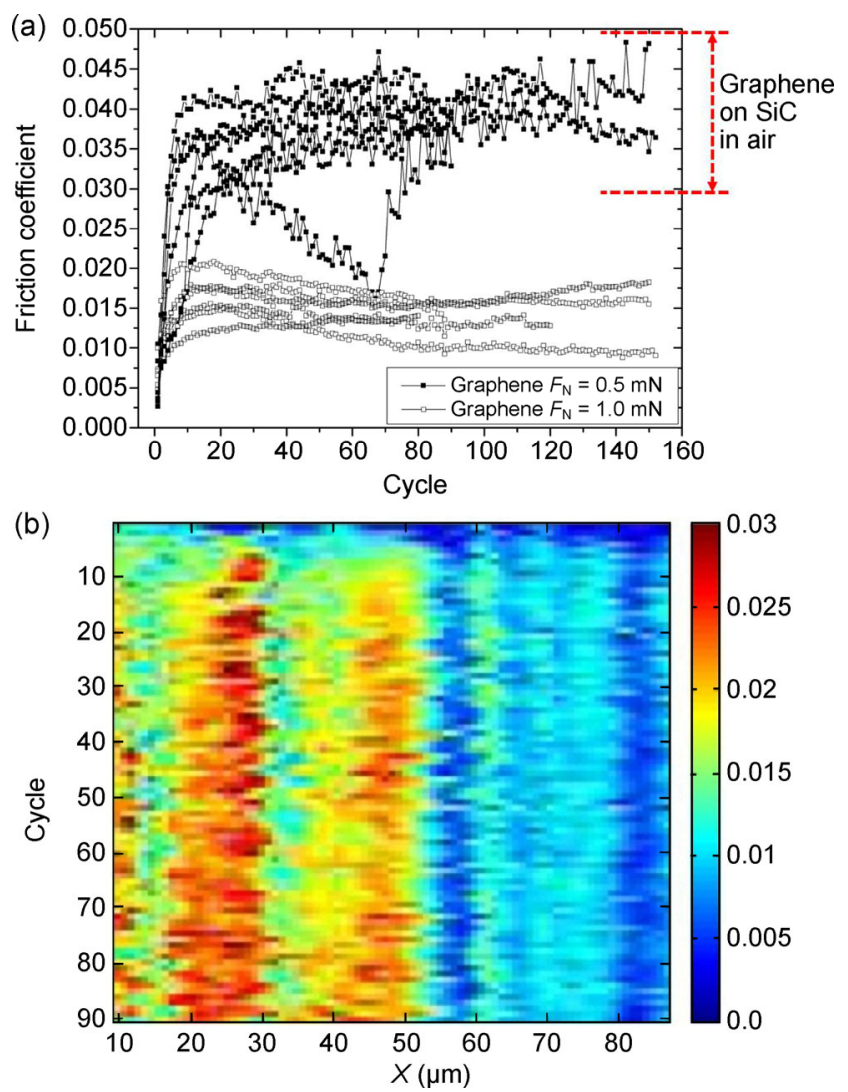

Fig. 3 (a) Friction coefficient of ruby against graphene grown on SiC. Tests were performed at two different loads: $0.5 \mathrm{mN}$ (black squares) and $1.0 \mathrm{mN}$ (empty squares). The red dashed lines on the right side of the graph show the range of the results obtained on the graphene measured in air in our previous work [20]; (b) Triboscopy map. Each data point represents a spot of about $0.1 \mu \mathrm{m}$ length. The data were recorded with an applied load of $1.0 \mathrm{mN}$. Big portions of the graphene layer maintain low friction along the entire experiment of 90 cycles. in the experiments in air [21] we can notice patches of graphene standing intact in the wear track for the entire measurements. However, a first difference with previous results is that patches are larger. In ambient conditions we found that areas were about 2 to $5 \mu \mathrm{m}$ large while here patches extend to almost $10 \mu \mathrm{m}$. Besides the size of the spots with unchanged friction coefficient also the density of these spots is higher. As can be seen in Fig. 3(b) the entire right side of the wear track maintained low friction and thus low wear along the entire experiment. Towards the end of the experiments, areas with low friction still span several tens of microns reducing the final average friction coefficient. The triboscopy map presented in Fig. 3(b) was obtained from one particular test but the reliability of the method is confirmed by the values reported in Fig. 3(a). Each data point plotted in Fig. 3(a) is obtained by averaging the values of a single cycle (one line of a triboscopy image). This means that the position of the patches or their dimension can slightly change from one test to the other (and the triboscopy will then be different), yet the average behavior is consistently pertained. This is underlined by the fact that all of the curves reported in the graph follow the same trend and are concentrated in a small range.

\subsection{Friction of graphene and of $\mathrm{SiC}$ as a function of sliding direction}

In order to explore and to compare possible friction anisotropy, the same measurements were performed in different directions with respect to the steps direction (see Fig. 4) both on $\mathrm{SiC}$ and on graphene. On SiC two directions marked as direction $\mathrm{A}$ (across the steps) and direction $B$ (along the steps) were investigated. The friction coefficient obtained sliding in direction A stays around 0.25 and is about 2 times higher than that obtained sliding along the steps $(\mu=0.1)$.

On graphene we performed tests in three different directions marked A, B and C. Direction A is approx. 45 degrees to the step edges, $B$ is perpendicular and $C$ is parallel to the edges. Along all of the three directions we obtained the same values of friction and the same behavior. After the first cycle at about 0.006 the friction coefficient rises to 0.011 and then it stays almost constant for the rest of the experiment. 

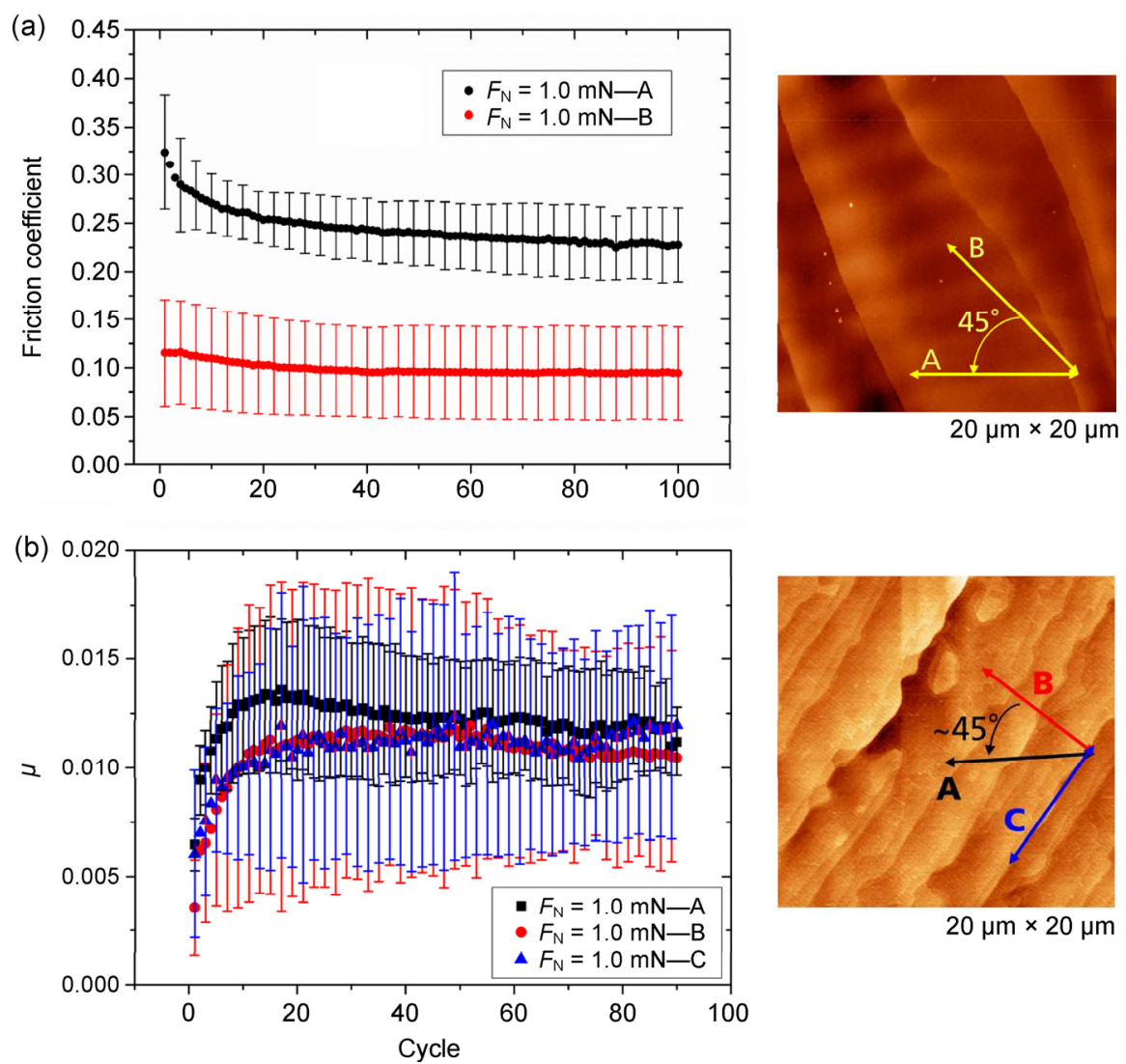

Fig. 4 Friction dependence on the sliding direction. (a) On SiC two different directions were analyzed as shown in the right side of the picture; (b) on graphene three sliding directions were measured.

A further analysis of the friction loops shows that steps of the substrate influence the interaction between the sphere and the sample surface (see Figs. 5 and 6).

Sliding on SiC (Fig. 5) in direction A, the oscillation in the friction force is around $10 \mu \mathrm{N}$ while in direction $\mathrm{B}$ there is no evidence of a periodical fluctuation. The amplitude of the oscillations is quite constant along one friction loop and the distance between two peaks is comparable with the terrace dimension of the $\mathrm{SiC}$ single crystal. It is interesting to notice that at cycle number 3 the peaks are not very clear because a water layer might still present on the surface, while at cycle 50 we find high oscillations. The reduction at cycle 100 is possibly due to wear of the surface.

On graphene (Fig. 6) some oscillations are still visible in the friction loops but they are not higher than $5 \mu \mathrm{N}$. Differently from $\mathrm{SiC}$, friction loops obtained on graphene show the same oscillation amplitude at cycle 3 and 20 and a lowering at the end of the measurement (cycle 100) due to wear.

\section{Discussion}

We showed that a ruby sphere sliding on single layer of epitaxial graphene leads to a friction coefficient that is even lower than that obtained in ambient conditions [21]. Therefore single-layer epitaxial graphene/SiC (0001) has the potential to excellently lubricate a tribocontact in the absence of air. In vacuum the friction coefficient reaches values of 0.01 after a start at 0.006 . The starting value is about three times lower than what is seen in air tests while the steady state value remains 5 times lower and does not show signs of deterioration after 100 cycles.

Since the large reduction of the friction coefficient of single-layer graphene in contrast to clean $\mathrm{SiC}$ was already discussed in our previous paper [21], here we want to focus on the differences between the friction in air and UHV. Indeed besides showing a strong friction reduction by graphene, we also noticed that the single layer of epitaxial graphene measured in 

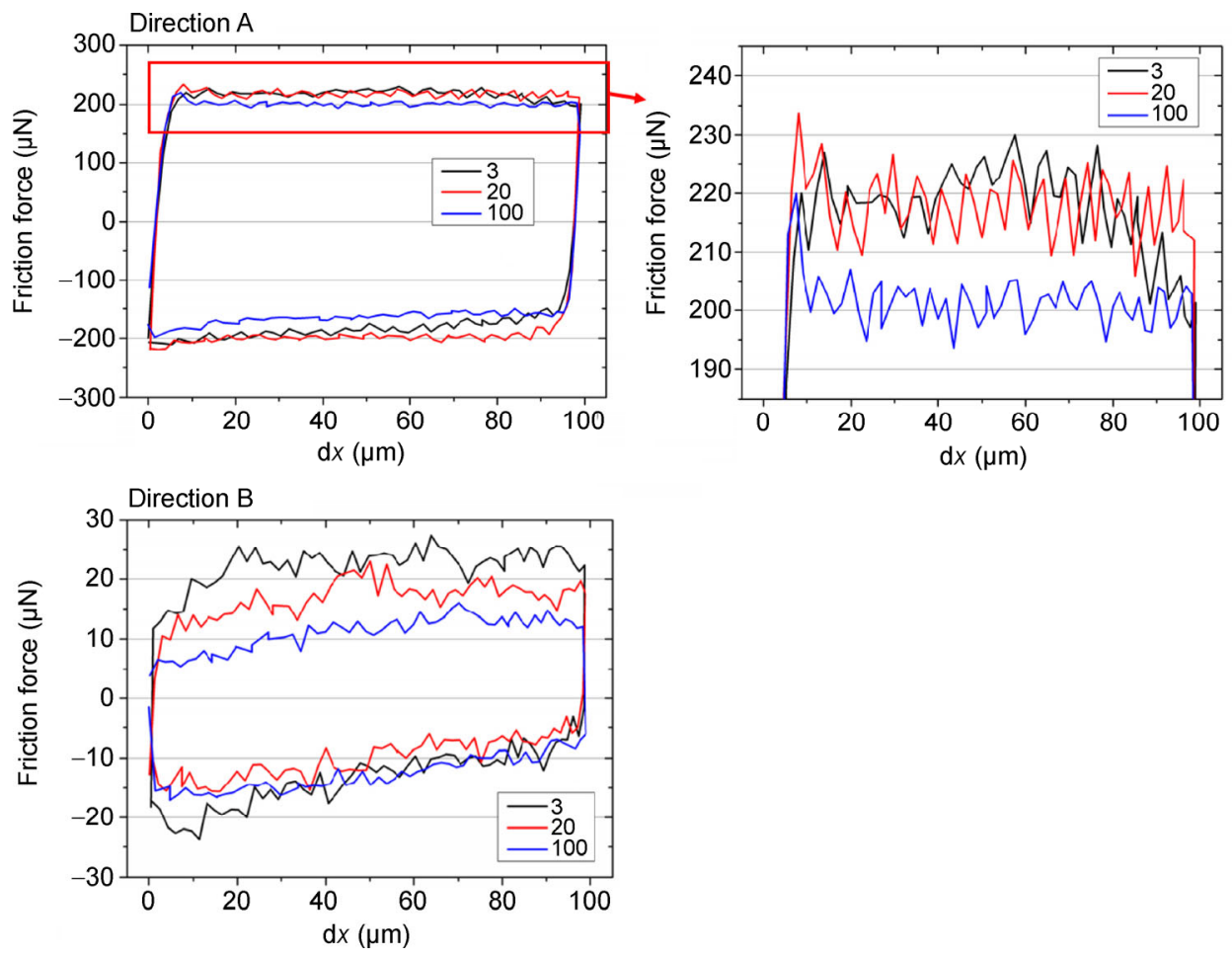

Fig. 5 Friction loops measured with a ruby sphere against $\mathrm{SiC}$ sliding in different directions $\mathrm{A}$ and $\mathrm{B}$ as showed in Fig. 4. Normal force $F_{\mathrm{N}}=0.5 \mathrm{mN}$. The three loops shown correspond to the $3^{\text {rd }}, 20^{\text {th }}$ and $100^{\text {th }}$ cycle. The right panel shows a zoom in of the friction loop in direction $\mathrm{A}$.
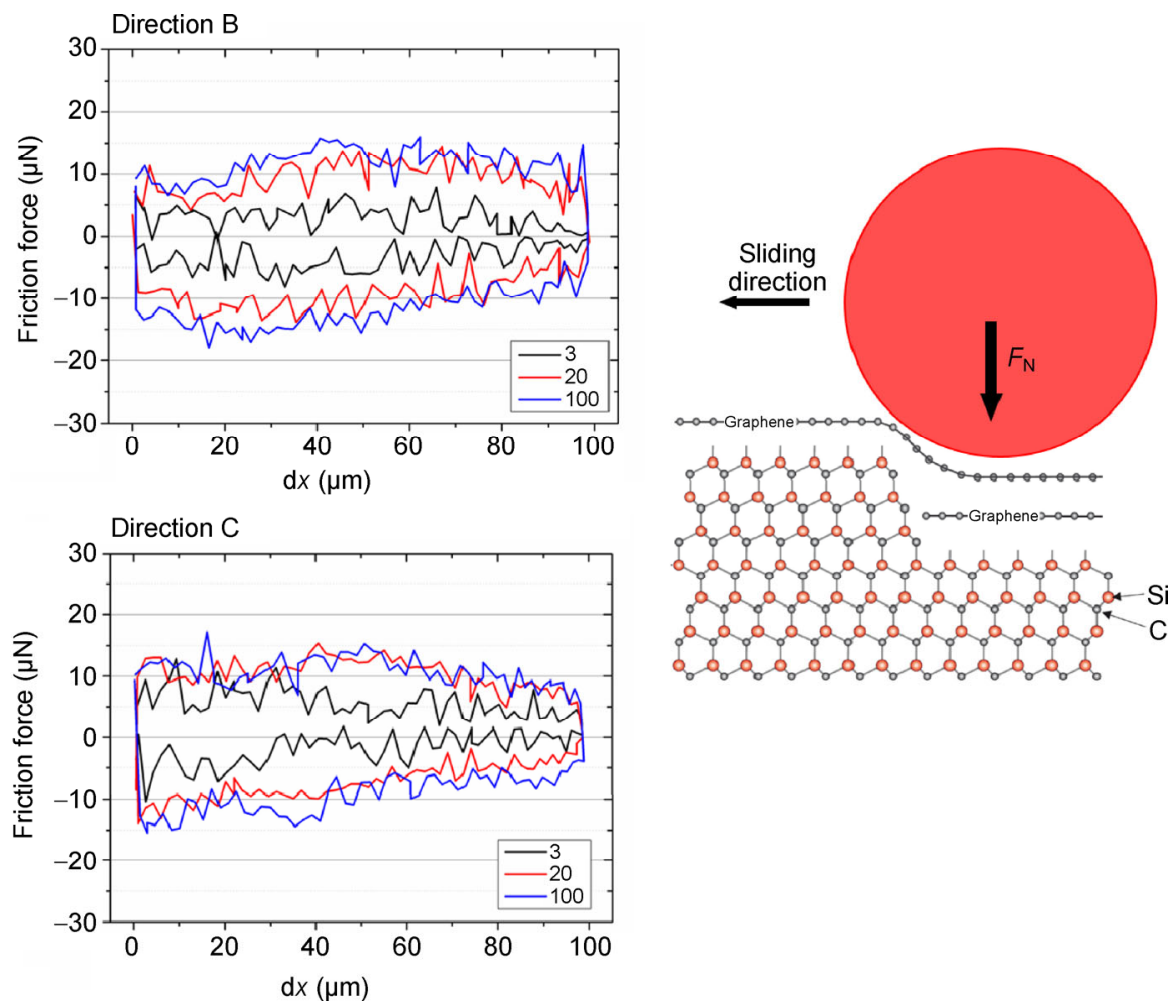

Fig. 6 Friction loops sliding with a ruby sphere on graphene in different directions $\mathrm{B}$ and $\mathrm{C}$ as showed in Fig. 4 . Normal force $F_{\mathrm{n}}=$ $0.5 \mathrm{mN}$. The three loops shown correspond to the $3^{\text {rd }}, 20^{\text {th }}$ and $100^{\text {th }}$ cycles. The right side shows a schematic of the contact to illustrate the "carpet effect" of graphene (illustration is reproduced from Bennewitz et al.). 
vacuum showed a slightly different behaviour than that observed in air. Instead of continuous increasingly values of the friction coefficient after a first running-in period in ambient air we observed that under UHV conditions $\mu$ stabilizes at a specific value after the running-in. This observation can be linked to the presence of the amount of remaining graphene patches. The triboscopy analysis clearly shows that the graphene layer gets worn along the 100 cycles. The damage is visible but it is limited if compared to the air tests. It is clear that graphene patches are larger than what we have previously seen in air [21]. At the same time damaged areas where the stable interface layer is exposed (left side of Fig. 3(b)) show a friction coefficient of 0.03 only. The above discussion of the results is based on measured triboscopy maps. Indeed it was not possible to locate the wear tracks after the experiments both because of the small sliding distance of the tribometer and because of the negligible wear of the SiC substrate. Because of this the search for the wear tracks by AFM failed and did not allow for topographical confirmation of the conclusions extracted from the triboscopy images. At the same time the results obtained in our previous work based both on triboscopy and AFM. The comparison of those experiments with the present work makes us quite confident on the statements we make in this paper.

We speculated in Ref. [21] that the initial running-in period visible in Figs. 2 and 3 can be explained by the high local shear stress produced by micro-asperities of the ruby sphere, cutting the graphene along steps of the $\mathrm{SiC}$ substrate and thereby removing flakes of the graphene film. Although the mean Hertzian pressure is quite low (few hundreds of MPa), the actual pressure at the asperity might reach local pressures in the range of Gigapascals. So the contact pressure ranges from few hundreds MPa (calculated with Hertz theory) to GPa in case of few small asperities [21].

After this first destructive period the remaining patches do not sustain any further damage. This could be the consequence of a higher stability of these patches due to a lack of defects and a stronger bonding to the substrate in that specific area. Also it might be possible that in ambient conditions oxygen or water is able to weaken the graphene films, which speeds up the wear of the film once a defect is created [26]. Further indications that the wear of the graphene layer is enhanced by tribochemical effects come from molecular dynamics (MD) simulations that have shown that even contact pressures of a few GPa should not break the graphene layer [27]. The running-in effect cannot be observed on $\mathrm{SiC}$ because the surface is not subjected to wear [21].

In the same way we believe the improvement of the friction of graphene with respect to the previous work is due to the lack of water molecules on the surface of the sample and of the pin. We speculate that the water layer that is known to usually form on surfaces in air is strongly reduced in vacuum and just a layer of physisorbed molecules can stick to the surface. These residual water molecules physisorbed to the surface would desorb during the very first scan and pumped away. This lack of water also induces the load dependency of friction both on bare SiC and on graphene.

The absence of other contaminants in the environment enhances the lubricating effect of graphene and maintains at a low value the friction coefficient of the interface layer. In fact the presence of a carbon buffer layer and its role in graphene lubrication were similarly discovered by Kim et al. for graphene grown on and transferred from a Ni substrate [15]. Therefore while the graphene layer provides extremely low friction coefficients for the intact surface, the stable interface layer assures the still favorable coefficient of friction after hundreds of sliding cycles.

Another clear difference between these tests and those showed in air is the presence of anisotropy of friction on $\mathrm{SiC}$ that disappears when graphene is present. The steps of $\mathrm{SiC}$ produce fluctuation of the friction force of 10 to $15 \mu \mathrm{N}$ (Fig. 6). This fluctuation is not visible on graphene in any direction (Fig. 5). We believe this is due to a "carpet effect" of graphene, meaning that the graphene layer masks the effect of the $\mathrm{SiC}$ steps. When the sphere slides on the surface graphene helps it in climbing up the steps. A similar effect was reported by Filleter and Bennewitz [14]. They studied the frictional properties of graphene on $\mathrm{SiC}$ by means of AFM and they noticed that the AFM silicon tip slides smoothly across a SiC step when this is covered by one or two layers of graphene (see Fig. 7 in Ref. [14]). 


\section{Conclusions}

To our knowledge this paper is the first to report the frictional properties of graphene in UHV at the microscale. The results were also compared with previous tests performed in air on the same kind of sample.

The lubrication properties shown in our previous work have been confirmed with this study in UHV. In fact in UHV graphene shows an even lower friction coefficient (three times lower in the best conditions). Although we assume that the mechanisms that bring to a lubricating effect are the same presented in air tests, it is surprising that the graphene layer behaves differently than bulk graphite. The lowering of the coefficient of friction could be due to a reduction of the water layer on the sample and on the counter body. Moreover the improved stability of the single-layer graphene in vacuum sheds light also on the wear mechanism in ambient conditions. Since the patches of graphene remaining on the wear track after the wear process show a much larger size compared to tests in air, the absence of oxygen and/or water could be the reason of the reduction in ambient conditions if we assume a tribochemical wear mechanism.

Finally anisotropy of the friction on $\mathrm{SiC}$ was seen. This effect is related to the presence of the terrace steps on the surface of $\mathrm{SiC}(0001)$ and it is masked by the presence of graphene. Indeed no anisotropy was found during the tests performed on $\mathrm{SiC}$ covered with graphene. This is explained with a "carpet behavior" of graphene.

\section{Acknowledgements}

We thank Thomas Seyller of the University of ErlangenNürnberg for samples preparation and acknowledge support from the Federal Ministry of Education and Research (BMBF No. 03X0107D). The authors would like to also acknowledge Professor R. Bennewitz for useful discussions.

Open Access: This article is distributed under the terms of the Creative Commons Attribution License which permits any use, distribution, and reproduction in any medium, provided the original author(s) and source are credited.

\section{References}

[1] Donnet C, Erdemir A. Solid lubricant coatings: Recent developments and future trends. Tribol Lett 17(3): 389-397 (2004)

[2] Ludema K C. In Friction, Wear, Lubrication: A Textbook in Tribology. Florida (US): CRC Press, Inc, 1993: 69-155.

[3] Lancaster J K. A review of the influence of environmental humidity and water on friction, lubrication and wear. Tribol Int 23(6): 371-389 (1990)

[4] Savage R H. Graphite lubrication. J Appl Phys 19: 1-10 (1948)

[5] Fusaro R L. Lubrication of space systems. Lubr Eng 3: 182-194 (1995)

[6] Singer I L. Solid lubrication processes. Fundamentals of friction: Macroscopic and microscopic processes. NATO ASI Series 220: 237-261 (1992)

[7] Stoyanov P, Strauss H W, Chromik R R. Scaling effects between micro- and macro-tribology for a Ti--MoS 2 coating. Wear 274-275: 149-161 (2012)

[8] Geim A K, Novoselov K S. The rise of graphene. Nat Mater 6: $183-191$ (2007)

[9] Yu M-F, Lourie O, Dyer M J, Moloni K, Kell T F, Ruoff R S. Strength and breaking mechanism of multiwalled carbon nanotubes under tensile load. Science 287(5453): 637-640 (2000)

[10] Lee C G, Wei X D, Kysar J W, Hone J. Measurement of the elastic properties and intrinsic strength of monolayer graphene. Science 321(5887): 385-388 (2008)

[11] Gao Y W, Hao P. Mechanical properties of monolayer graphene under tensile and compressive loading. Physica $E$ 41(8):1561-1566 (2009)

[12] Lee C, Wei X, Li Q, Carpick R, Kysar J W, Hone J. Elastic and frictional properties of graphene. Physica Status Solidi B 246(11-12): 2562-2567 (2009)

[13] Schwarz U D, Zworner O, Koster P, Wiesendanger R. Quantitative analysis of the frictional properties of solid materials at low loads. Phys Rev B 56: 6987-6996 (1997)

[14] Filleter T, Bennewitz R. Structural and frictional properties of graphene films on $\mathrm{SiC}$ (0001) studied by atomic force microscopy. Phys Rev B 81: 155412 (2010)

[15] Kim K, Lee H J, Lee C, Lee S K, Jang H, Ahn J H, Kim J H, Lee H J. Chemical vapor deposition-grown graphene: The thinnest solid lubricant. ACS Nano 5: 5107-5114 (2011)

[16] Lee C, Li Q, Kalb W, Liu X, Berger H, Carpick R W, Hone J. Frictional characteristics of atomically thin sheets. Science 328: 76-80 (2010)

[17] Berman D, Erdemir A, Sumant A V. Few layer graphene to reduce wear and friction on sliding steel surfaces. Carbon 54: 454-459 (2013) 
[18] Berman D, Erdemir A, Sumant A V. Reduced wear and friction enabled by graphene layers on sliding steel surfaces in dry nitrogen. Carbon 59: 167-175 (2013)

[19] Filleter T, McChesney J L, Bostwick A, Rotenberg E, Emtsev K V, Seyller T H, Horn K, Bennewitz R. Friction and dissipation in epitaxial graphene films. Phys Rev Lett 102: 086102 (2009)

[20] Washizu H, Kajita S, Tohyama M, Ohmori T, Nishino N, Teranishi H, Suzuki A. Mechanism of ultra low friction of multilayer graphene studied by coarse-grained molecular simulation. Faraday Discuss 156: 279-291 (2012)

[21] Marchetto D, Held C, Hausen F, Wählisch F, Dienwiebel M, Bennewitz R. Friction and wear on single-layer epitaxial graphene in multi-asperity contacts. Tribol Lett 48: 77-82 (2012)

[22] Marchetto D, Benzig R, Korres S, Dienwiebel M. Design and testing of an ultra high vacuum microtribometer. Tribology-Materials, Surfaces \& Interfaces 6: 95-101 (2012)

[23] Emtsev K V, Bostwick A, Horn K, Jobst J, Kellogg G, Ley L, McChesney J L, Ohta T, Reshanov S A, Röhrl J, Rotenberg E,

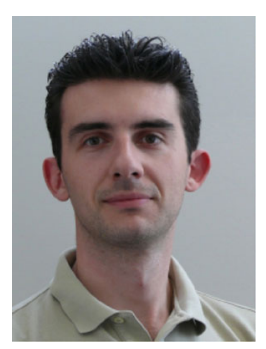

Diego MARCHETTO. $\mathrm{He}$ is a researcher and obtained his master degree and $\mathrm{PhD}$ degree in physics in 2003 and 2010 from University of Modena and Reggio Emilia (Italy). He worked with Dr. Martin Dienwiebel as a post doctoral fellow at Fraunhofer Institute in Germany

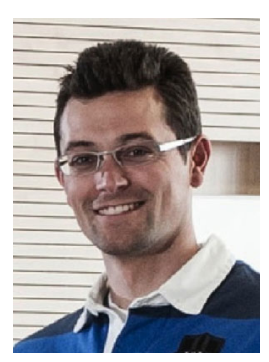

Tim FESER. He obtained his master degree and $\mathrm{PhD}$ degree in mechanical engineering at the

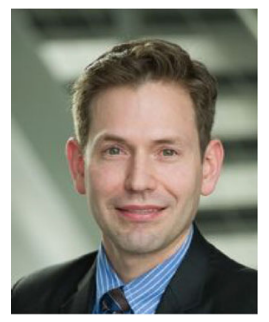

Martin DIENWIEBEL. He is an assistant professor and obtained his master degree in physics in 1997 from Bonn University, Germany, and received his $\mathrm{PhD}$ degree in 2003 from Leiden University, The Netherlands. During this period he was working as visiting scientist at the Tokyo Institute of Technology, Japan. From 2003-2007 he
Schmid A K, Waldmann D, Weber H B, Seyller T. Towards wafer-size graphene layers by atmospheric pressure graphitization of silicon carbide. Nat Mater 8: 203-207 (2009)

[24] Lauffer P, Emtsev K V, Graupner R, Seyller T, Ley L, Reshanov S A, Weber H B. Atomic and electronic structure of few-layer graphene on $\mathrm{SiC}$ (0001) studied with scanning tunneling microscopy and spectroscopy. Phys Rev B 77: 155426 (2008)

[25] Wählisch F, Hoth J, Held C, Seyller T, Bennewitz R. Friction and atomic-layer-scale wear of graphitic lubricants on $\mathrm{SiC}$ (0001) in dry sliding. Wear 300(1-2): 78-81 (2013)

[26] Moras G, Pastewka L, Walter M, Schnagl J, Gumbsch P, Moseler M. Progressive shortening of sp-hybridized carbon chains through oxygen-induced cleavage. J Phys Chem C 115(50): 24653-24661 (2011)

[27] Klemenz A, Pastewka L, Balakrishna S G, Caron A, Bennewitz R, Moseler M. Atomic scale mechanisms of friction reduction and wear protection by graphene. Nano Lett 14(12): 7145-7152 (2014)

from 2009-2014. Presently he works as researcher at the University of Modena and Reggio Emilia (Italy). Currently his research areas include tribology of graphene, microscale friction and lubrication at low temperature. He has participated in many research projects and has published more than 10 papers on international journals.

Karlsruhe Institute of Technology in 2009 and 2013. He is presently working at BASF SE, Ludwigshafen, Germany.

worked at the Tribology and Research department of IAVF Antriebstechnik AG, Germany. In 2008 he received an Emmy-Noether fellowship of the Deutsche Forschungsgemeinschaft. Presently he is working as group leader at Fraunhofer Institute of Technology and Karlsruhe Institute of Technology. His research interests are in the area of superlow friction, runningin, superlubricity, analytical and nano-scale processes of tribological interfaces. 